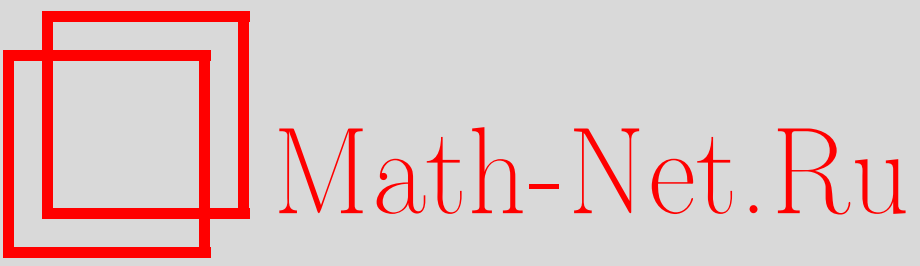

Д. В. Гугнин, О непрерывных и неприводимых $n$-гомоморфизмах Фробениуса, УМН, 2005, том 60, выпуск 5, 163-164

DOI: https://doi.org/10.4213/rm1645

Использование Общероссийского математического портала Math-Net.Ru подразумевает, что вы прочитали и согласны с пользовательским соглашением

http://www.mathnet.ru/rus/agreement

Параметры загрузки:

IP: 54.237 .206 .68

26 апреля 2023 г., 16:04:28 


\title{
О НЕПРЕРЫВНЫХ И НЕПРИВОДИМЫХ $n$-ГОМОМОРФИЗМАХ ФРОБЕНИУСА
}

\author{
Д.В. Гугнин
}

Понятие $n$-алгебраического отображения было введено в [1], эквивалентное ему понятие $n$-гомоморфизма Фробениуса появилось в [2]. Теория развита в [3], [4]. Близкие к $n$-гомоморфизму понятия - псевдохарактер [5] и псевдопредставление [6]. Далее под алгеброй мы понимаем коммутативную, ассоциативную алгебру с единицей над полем комплексных чисел $\mathbb{C}$.

Пусть $f: A \rightarrow B$ - линейное отображение алгебр. Для любого натурального $k$ определим полилинейные симметричные отображения $\Phi_{k}(f): A^{k} \rightarrow B$. Запишем перестановку $\sigma \in \Sigma_{k}$ в виде произведения попарно непересекающихся циклов $\gamma_{1} \cdots \gamma_{r}$, включая и циклы длины 1 . Положим $f_{\gamma}\left(a_{1}, \ldots, a_{k}\right)=f\left(a_{i_{1}} \cdots a_{i_{q}}\right)$ для цикла $\gamma=\left(i_{1}, \ldots, i_{q}\right)$ и $f_{\sigma}=f_{\gamma_{1}} \cdots f_{\gamma_{r}}$. Определим $\Phi_{k}(f)$ формулой:

$$
\Phi_{k}(f)\left(a_{1}, \ldots, a_{k}\right)=\sum_{\sigma \in \Sigma_{k}} \operatorname{sign}(\sigma) f_{\sigma}\left(a_{1}, \ldots, a_{k}\right) .
$$

ОПРЕДЕЛЕниЕ 1 . Линейное отображение $f: A \rightarrow B$ назьвается $n$-гомоморфизмом Фробениуса (кратко, $n$-гомоморфизмом), если $\Phi_{n+1}(f) \equiv 0$ и $f(1)=n$.

ЗАмЕчАНИЕ. 1-гомоморфизм - это обычный гомоморфизм алгебр.

Из определения 1 легко следует, что композиция 1-гомоморфизма и $n$-гомоморфизма в любом порядке дает снова $n$-гомоморфизм.

Лемма 1. Пусть $f: A \rightarrow B-n$-гомоморфизм $u \pi: A \rightarrow D-$ эпиморфизм алгебр. Если существует отображение $g: D \rightarrow B$ такое, что $f=g \pi$, то $g$ - однозначно определенный $n$-гомоморфизм.

Пусть $A$ - алгебра, $L \subset A$ - произвольное собственное подпространство. Введем следующий идеал: $\operatorname{MaxId}(L)=\{a \in A \mid a \cdot x \in L, \forall x \in A\}$. Легко видеть, что он является наибольшим среди всех идеалов, лежащих в $L$. Пусть $f: A \rightarrow B-n$-гомоморфизм, $\mathfrak{J}$ - идеал в $A, \mathfrak{J} \subset \operatorname{ker} f$. Тогда $f=g \pi$ в силу леммы 1 , где $\pi: A \rightarrow A / \mathfrak{J}$ - каноническая проекция и $g: A / \mathfrak{J} \rightarrow B$ однозначно определенный $n$-гомоморфизм.

Tеорема 1. Пусть $B$ - алгебра без нильпотентов и $f: A \rightarrow B-n$-гомоморфизм. Тогда $\operatorname{MaxId}(\operatorname{ker} f)=\left\{a \in A \mid f(a)=f\left(a^{2}\right)=\cdots=f\left(a^{n}\right)=0\right\}$.

СледСтвиЕ 1. Пусть $f: A \rightarrow \mathbb{C}$ некоторый $n$-гомоморфизм. Тогда выполнено неравенство соdim $\operatorname{MaxId}(\operatorname{ker} f) \leqslant n$.

Как показано в [3], сумма $n$ - и $m$-гомоморфизмов является $(n+m)$-гомоморфиизом.

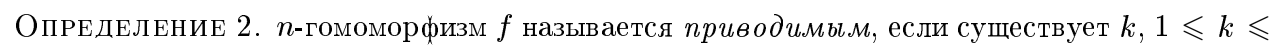
$n-1$, такое, что $f=g+h$, где $g$ и $h-k$ - и $(n-k)$-гомоморфизмы соответственно, иначе $f$ называется неприводимым. В частности, любой 1-гомоморфизм неприводим.

В работе [3] было доказано что любой $n$-гомоморфизм конечнопорожденной алгебры $A$ в $\mathbb{C}$ однозначно разлагается в сумму 1-гомоморфизмов. С помощью леммы 1 и следствия 1 мы распространяем этот резултат на случай произвольной алгебры $A$.

Теорема 2. Пусть $f: A \rightarrow \mathbb{C}-n$-гомоморфизм. Тогда существуют и единственны с точностью до перестановок 1-гомоморфизмы $f_{1}, \ldots, f_{n}: A \rightarrow \mathbb{C}$ такие, что $f=f_{1}+$ $\cdots+f_{n}$.

Пусть $X, Y$ - компактные хаусдорфовы пространства. В алгебрах непрерывных комплекснозначных функций $\mathscr{C}(X)$ и $\mathscr{C}(Y)$ рассматриваем sup-норму $\left(\|f\|=\max _{x \in X}|f(x)|\right)$ и соответствующую ей топологию. 
ТЕОРема 3. Любой $n$-гомоморфизм $F: \mathscr{C}(X) \rightarrow \mathscr{C}(Y)$ непрерывен и его норма равна $n$.

Структура всех непрерьвных $n$-гомоморфизмов $F: \mathscr{C}(X) \rightarrow \mathscr{C}(Y)$ описана в [7] и имеет следующий вид. Обозначим через $\Phi_{n}(\mathscr{C}(X), \mathscr{C}(Y))$ множество непрерывных $n$-гомоморфизмов из $\mathscr{C}(X)$ в $\mathscr{C}(Y)$. Пусть $S y m^{n} X=X^{n} / \Sigma_{n}-n$-я симметрическая степень пространства $X$. Точки $S_{y m}{ }^{n} X$ - это неупорядоченные наборы $n$ точек из $X:\left[x_{1}, \ldots, x_{n}\right]=\left[x_{\sigma(1)}, \ldots, x_{\sigma(n)}\right]$, $\forall \sigma \in \Sigma_{n}$. Существует каноническая биекция

$$
\begin{gathered}
\Psi: \mathscr{C}\left(Y, \operatorname{Sym}^{n} X\right) \rightarrow \Phi_{n}(\mathscr{C}(X), \mathscr{C}(Y)), \quad[(\Psi G) f] y=f\left(G_{1}(y)\right)+\cdots+f\left(G_{n}(y)\right), \\
\forall G \in \mathscr{C}\left(Y, \operatorname{Sym}^{n} X\right), \forall f \in \mathscr{C}(X), \forall y \in Y, G(y)=\left[G_{1}(y), \ldots, G_{n}(y)\right] \in \operatorname{Sym}^{n} X
\end{gathered}
$$

Кроме того, мы показали, что $\Psi$ есть гомеоморфизм, если в $\mathscr{C}\left(Y, S y m^{n} X\right)$ рассматривается компактно-открытая топология, а в $\Phi_{n}(\mathscr{C}(X), \mathscr{C}(Y)) \subset \mathscr{L}(\mathscr{C}(X), \mathscr{C}(Y))$ топология поточечной сходимости.

ОПРЕДЕЛЕНИЕ 3 . Пусть $X$ и $Y$ - топологические пространства. Скажем, что $X$ mопологически содерэит $Y$, если в $X$ существует подпространство $Z$, гомеоморфное $Y$.

Теорема 4. Пусть $X$ и $Y$ - компактные хаусдорфовы пространства, $X$ топологически содержит букет трех отрезков, $Y$ топологически содержит окружность. Тогда для любого $n$ существует бесконечно много неприводимых $n$-гомоморфизмов $F: \mathscr{C}(X) \rightarrow \mathscr{C}(Y)$.

В заключение приведем одно построение и вытекающий из него результат. Зафиксируем алгебру $A$. Введем следующую категорию $\mathscr{F}_{n}(A)$. Объектами $\mathscr{F} n(A)$ служат пары $(B, f)$, где $B$ - алгебра, $f: A \rightarrow B-n$-гомоморфизм. Морфизм $\pi$ из $(B, f)$ в $(C, g)$ - это 1-гомоморфизм из $B$ в $C$ такой, что $g=\pi f$. Обозначим через $S^{n} A$ симметрическую подалгебру в $A^{\otimes n}$. Назовем универсальным $n$-гомоморфизмом алгебры $A$ отображение $\chi_{n}(A): A \rightarrow S^{n} A$, $\chi_{n}(A)(a)=a \otimes 1 \otimes \cdots \otimes 1+\cdots+1 \otimes \cdots \otimes 1 \otimes a$. Следующая лемма по существу содержится в [3] и [4].

ЛЕмма 2. Пара $\left(S^{n} A, \chi_{n}(A)\right)$ является универсальным отталкивающим обвектом в $\mathscr{F}_{n}(A)$.

Теорема о композиции $n$-гомоморфизмов доказана в [7] в предположении, что $C$ - алгебра функций. С помощью леммы 2 мы снимаем это условие.

Tеорема 5. Пусть $f: A \rightarrow B-n$-гомоморфизм, $g: B \rightarrow C-m$-гомоморфизм. Тогда $g \circ f: A \rightarrow C-n m$-гомоморфизм.

Я хочу поблагодарить моего научного руководителя профессора В.М. Бухштабера за постановку задач, многочисленные полезные обсуждения и постоянное внимание к моей работе.

\section{СПИСОК ЛИТЕРАТУРЫ}

[1] В. М. Бухштабер, Е. Г. Рис // УМН. 1996. Т. 51. № 4. С. 149-150. [2] В. М. Бухштабер, Е.Г. Рис // УМН. 1997. Т. 52. №2. С. 159-160. [3] V.M. Buchstaber, E. G. Rees // Selecta Math. (N.S.). 2002. V. 8. №4. P. 523-535. [4] B. M. Бухштабер, Е. Г. Рис // УМН. 2004. Т. 59. №1. С. 125-144. [5] R. Rouquier // J. Algebra. 1996. V. 180. № 2. P. 571-586. [6] R. L. Taylor // Duke Maths. J. 1991. V. 63. № 2. P. 281-332. [7] V. M. Buchstaber, E. G. Rees. Frobenius $n$-homomorphisms and branched coverings // www.maths.ed.ac.uk/ elmer.

Московский государственньй университет им. М. В. Ломоносова
Представлено В. М. Бухштабером Принято редколлегией 07.09.2005 